\title{
Added Value of MRI over CT of the Brain in Intensive Care Unit Patients
}

\author{
Haifa M. Algethamy, Mohamed Alzawahmah, G. Bryan Young, Seyed M. Mirsattari
}

\begin{abstract}
Background: Intensive care unit (ICU) patients with neurological impairments often require neuroimaging. However, the relative sensitivity of various imaging modalities of the brain has not yet been explored in this population. Methods: In this study, we compare the findings of CT and MRI scans in ICU patients to (1) identify the number and rate of clinically relevant lesion detected by MRI while missed by CT and vice versa and (2) determine specific lesion types for which CT versus MRI discrepancies exist. A review of medical records included CT and MRI reports of patients who underwent these procedures while they were patients in our ICUs between July 2004 and July 2009. MRI and CT were compared regarding their ability to detect clinically relevant abnormalities. Odds ratios with 95\% confidence limits were calculated to compare diagnostic categories regarding the rate of discrepant MRI versus CT findings, followed by power analyses to estimate sample sizes necessary to allow for further testing in a larger trial. Results: MRI revealed clinically relevant additional abnormalities over CT in 129 of 136 patients (95\%) that included the detection of additional finding for $15 / 27$ hemorrhagic lesions (55.6\%), 33/36 (92\%) ischemic strokes, 19/27 (70\%) traumatic lesions, 8/14 (57\%) infections, 15/24 (62.5\%) metabolic abnormalities, and all seven neoplasms. Odds ratio analysis revealed the added sensitivity of MRI to be greater for ischemic and neoplastic lesions than for trauma, metabolic-related abnormalities, infection, or hemorrhage. Conclusions: MRI is more sensitive than CT in identifying clinically meaningful lesions in at least a subset of ICU patients, regardless of pathology.
\end{abstract}

RÉSUMÉ: Valeur ajoutée de l'IRM par rapport au CT du cerveau chez les patients hospitalisés à l'unité de soins intensifs. Contexte : Chez les patients présentant une atteinte neurologique, hospitalisés à l'unité de soins intensifs (USI), il est souvent nécessaire d'avoir recours à la neuroimagerie. Cependant, la sensibilité relative de différentes modalités d'imagerie du cerveau n'a encore jamais été explorée dans cette population de patients. Méthode : Dans cette étude, nous avons comparé les constatations aux scans CT et IRM chez des patients de l'USI afin d'identifier le nombre et le taux de lésions significatives au point de vue clinique détectées par l'IRM mais manquées par le CT et vice versa et de déterminer le type de lésions pour lesquelles il existe des divergences entre le CT et l'IRM. Nous avons effectué une revue des dossiers médicaux des patients qui avaient subi ces examens au cours de leur hospitalisation à l'USI entre juillet 2004 et juillet 2009, pour lesquels il y avait un rapport de CT et d'IRM au dossier. Nous avons comparé la capacité de l'IRM et celle du CT à détecter des anomalies significatives au point de vue clinique. Nous avons calculé les rapports de cotes avec un intervalle de confiance à $95 \%$ pour comparer le taux de divergence des constatations à l'IRM par rapport au CT selon les catégories de diagnostiques et nous avons réalisé des analyses de puissance pour estimer les tailles d'échantillons nécessaires en vue de poursuivre l'étude lors d'un essai de plus grande taille. Résultats : L'IRM a révélé des anomalies additionnelles significatives par rapport au CT chez 129/136 (95\%) patients incluant la détection de constatations additionnelles chez 15/27 (55,6\%) cas de lésions hémorragiques, 33/36 (92\%) cas d'accidents vasculaires cérébraux ischémiques, 19/27 (70\%) cas de lésions traumatiques, $8 / 14$ (57\%) cas d'infections, 15/24 (62,5\%) cas d'anomalies métaboliques et chacun des 7 cas de néoplasies. L'analyse des rapports de cotes a montré que la plus grande sensibilité de l'IRM en faisait un examen supérieur pour les lésions ischémiques et les lésions néoplasiques que pour les lésions traumatiques, les anomalies métaboliques, les infections ou les hémorragies. Conclusions : L'IRM est plus sensible que le CT pour identifier des lésions significatives au point de vue clinique chez au moins un sous-groupe de patients de l'USI, quelle que soit la pathologie en cause.

Keywords: Central nervous system pathology, computed tomography, intensive care unit patients, magnetic resonance imaging, prognosis, unresponsive patients

doi:10.1017/cjn.2015.52

Can J Neurol Sci 2015; 42: 324-332

\section{INTRODUCTION}

In intensive care unit (ICU) patients with central nervous system (CNS) pathology, a detailed neurological examination is often impossible. One common reason for this is that the patient has a significantly decreased level of consciousness, sometimes to the point of being unresponsive. This can be caused either by the pathological process itself or by induced patient sedation and/or neuromuscular paralysis. In this sizeable patient population, therefore, clinical examination is of utmost importance, but provides incomplete assessment of underlying pathology, such that neuroimaging studies often are relied upon. The purposes of such imaging include identifying or confirming diagnoses, determining the extent of disease or injury, estimating prognosis,

From the Anesthesia and Critical Care Department, Faculty of Medicine, King Abdulaziz University, King Abdulaziz University Hospital, Jeddah, Saudi Arabia (HMA), Department of Clinical Neurological Sciences, Riyadh Military Hospital, Riyadh, Saudi Arabia (MA), Department of Clinical Neurological Sciences, Medical Biophysics,

Medical Imaging, and Psychology, London Health Sciences Centre, Western University, London, Ontario, Canada (GBA, SMM)

Received July 13, 2014. Final Revisions Submitted March 3, 2015.

Correspondence to: Haifa M Algethamy, Anesthesia and Critical Care Department, King Abdulaziz University Hospital, King Abdulaziz University, P.O. Box 80215, Jeddah 21589, Saudi Arabia. Email: halgethamy2020@gmail.com 
guiding management and, sometimes, making a decision regarding the withdrawal of aggressive treatment.

When imaging modalities have been compared, research has consistently demonstrated that MRI is superior to CT in many acute neuropathology settings, such as documenting brain trauma, ${ }^{1-4}$ intracranial hemorrhages, ${ }^{4-8}$ clinically apparent and silent brain infarcts, ${ }^{9-14}$ tumors, ${ }^{15,16}$ and encephalopathy. ${ }^{17}$ However, for decades, traditional ICU patient management protocols have called for CT images of the brain first, with further imaging, such as MRI, reserved for cases of unexpected negative or inconclusive CT results. Advantages of CT over MRI are its cost, which generally is less than half that of MRI, ${ }^{18}$ as well as its speed, easier access, and ability to accommodate patients who are either too agitated or too unresponsive to remain still. ${ }^{4,19}$

This being said, there are also potential costs of delay, especially in critically ill patients with CNS pathology in whom it often is crucial for a correct diagnosis to be made and appropriate aggressive therapy instituted. In fact, errors or delays in diagnosis may adversely affect patient outcomes, with potential dire consequences. This, in turn, may prolong time in the ICU, overall hospitalization, and subsequent health care costs if CT scan ordered before an MRI.

Although this has not been studied in the ICU setting, aggressive imaging including MRI in the early stages of management has already been shown to both enhance outcomes and reduce costs in patients with other neurological disorders such as dementia. ${ }^{20}$ The current study is retrospective and determines if there is any need to further study this issue within the neurocritical care setting. More specifically, we sought to systematically compare the findings of CT and MRI scans in ICU patients to (1) identify the number of clinically relevant lesions detected by MRI while missed by CT and vice versa; (2) determine specific lesion types for which CT versus MRI discrepancies exist; and (3) estimate the number of patients who would be necessary to compare diagnostic groups and perform further inferential testing within the context of a larger clinical trial. Our main a priori hypotheses were (1) that some clinically relevant finding would be identified on MRI that had been missed on CT in a sizeable percentage (more than 50\%) of patients; (2) that the reverse would not be true; and (3) that a larger clinical trial is both warranted and feasible. Should these three hypotheses prove to be accurate, the next step for further study will be to design a multicentre study to prospectively compare CT and MRI findings in ICU patients with CNS pathology and decreased consciousness with respect to (1) diagnostic yield, (2) effect on actual management (i.e. how often is management altered by a finding on MRI not seen on CT), (3) mortality and morbidity rates, and (4) costs via a detailed estimation of direct health care costs. If the current study identifies specific lesion types for which MRI identifies lesions missed by CT in the majority of cases, these lesion types will set the patient population of interest for the subsequent, intended prospective study.

\section{MeTHODS}

Before any data collection, the current study had been approved by the University of Western Ontario Ethics Review Board for Human and Animal Studies.

The study was retrospective and observational, with limited intention to perform inferential analysis. As stated previously, our goals were (1) to identify the percentage of patients for whom lesions seen on MRI were either missed or underestimated in magnitude/severity on CT and vice versa; (2) to identify specific lesions types for which this was most evident; and (3) to calculate sample size requirements for a larger group comparison study.

As a first step, all adult patients (age 18 and older) were identified who (1) had been admitted to either the medical, surgical, or trauma ICUs at either of the two campuses (University Hospital and Victoria Hospital) of the London Health Sciences Centre between July 2004 and July 2009 and (2) had undergone either CT or MRI of the brain while a patient in the ICU. As a second step, we identified that subset of patients who had undergone both a CT and MRI of the brain within 30 days of each other.

We then retrospectively and systematically accessed and reviewed all CT and MRI reports from those patients as well as all electronic and written medical records for each patient over the course of the incident hospitalization. From these records, we identified patient demographic (age, gender) and clinical characteristics, including the reason for ICU admission and the timing and indications for neuroimaging.

Over that 5-year span, 140 patients were identified who had both a brain CT and MRI scans within 30 days of each other. Of this number, two were excluded because their CT scans were not available for comparison to MRI. Two others were excluded because their MRIs had been ordered for some different indication than their CT scans - one patient's CT was performed preoperatively, whereas the MRI was done to assess postoperative changes; meanwhile, the other patient's clinical condition had changed between the two procedures. This left 136 subjects for final analysis.

Patient ages ranged from 21 to 86 years, with $67 \%$ being male. The reason for ICU admission was medical in $65 \%$ of cases, with surgery and trauma accounting for $15 \%$ and $20 \%$ of the patients, respectively. All patients had at least one CT scan before the MRI scan. For our analysis, the most recent CT scan prior to the MRI was used for comparison against the MRI scan.

MRI scans were obtained within 4-96 hours of the most recent CT scan in $99(73 \%)$ patients. Of the remaining 37 patients, 36 underwent MRI between 5 and 19 days after the most recent CT scan, whereas one patient underwent MRI 29 days after the last CT scan. All CT and MRI examinations were reviewed and reported upon by qualified neuroradiologists who had been provided with the patients' relevant clinical data. All CT and MRI examinations also were reviewed by the study investigator (M.A., a qualified stroke neurologist) for this study.

The CT and MRI scans were evaluated, as appropriate, for contusion, intra- or extra-axial hemorrhage, ischemia or infarction, diffuse axonal injury, and diffusion and/or perfusion abnormalities.

Based upon the diagnoses identified by CT/MRI findings, patients were categorized into six diagnostic groups: (1) hemorrhage; (2) ischemia; (3) trauma; (4) CNS infections; (5) metabolic abnormalities; and (6) neoplasms.

\section{Data Analysis}

Descriptive statistics are presented as percentages, with $95 \%$ confidence intervals calculated using Wilson's procedure corrected for continuity. ${ }^{21}$ Pearson $\chi 2$ analysis was performed to determine if differences existed across the six diagnostic groups with respect to the percentage of cases with clinically relevant MRI findings not detected on CT. Post hoc analysis was performed by calculating 
odds ratios with $95 \%$ confidence limits for each diagnostic pair. All inferential testing was two-tailed, and confidence intervals excluding 1.00 were considered statistically significant.

Power analysis then was performed using the identified rates for each of the six diagnostic categories to determine the number of subjects who would need to be enrolled to identify at least 30 patients with MRI findings not observed on CT, a number selected to allow for subgroup estimates of direct costs with $95 \%$ confidence limits within roughly $\pm 10 \%$. Power analysis also was performed over the entire sample to determine the size of the patient sample required to identify 30 patients for whom management was significantly altered, with a significant alteration defined as either (1) a new diagnosis requiring different management, (2) a specific treatment initiated that would not have been initiated based upon CT findings, or (3) withdrawal of medical support. For all sample size estimates, a $25 \%$ dropout rate was assumed, allowing for early patient mortality or other loss, such as transfer to another hospital.

\section{RESULTS}

In seven patients, both the CT and MRI scans were deemed normal, whereas abnormalities were detected in either the CT or MRI, or both, in each of the remaining 129 (95\%) patients.

Overall, clinically significant abnormalities were detected in 96 of the 136 CT scans (sensitivity $=70.6 \%$ ). In no instance was a lesion detected on CT that was not detected by MRI. Similarly, in no instance was a finding detected on CT that provided additional clinical insights beyond findings detected on MRI.

Conversely, abnormalities were apparent in 129 of the 136 MRI scans (94.9\%). In addition, MRI revealed additional pathology and/or more clearly delineated findings than those identified on CT for 85 patients $(63 \%)$, and changed the working diagnosis (initially based on CT scans and clinical examinations) for six $(4.4 \%)$ patients.

With respect to specific lesion types, MRI revealed additional abnormalities relevant to the patient's clinical status for 97 of 135 clinically relevant lesions ( $72 \%$ ), with some of the 129 patients having more than one lesion. This included the detection of additional findings for 15/27 hemorrhagic lesions (55.6\%), 33/36 (92\%) ischemic lesions, 19/27 (70\%) traumatic lesions, 8/14 (57\%) infections, 15/24 (62.5\%) metabolic abnormalities, and all seven neoplasms (Figure 1).

More specifically, by lesion type, in 33 of the 36 (92\%) patients with an ischemic lesion or lesions, the MRI either showed ischemic lesions that were not seen on CT (four patients) or additional details about lesions observed on CT-such as extension of the ischemic lesions, multiple territory cerebral microinfarcts, or brain stem lesions (29 patients). In 19 of 27 (70\%) patients with traumatic lesions, the MRI was more sensitive than CT with respect to the detection and delineation of the extent of the traumatic brain injury. In patients with a CNS infection, the MRI was superior to CT scans at detecting areas of focal cerebritis and demonstrating contrast enhancement of either the parenchyma or meninges. And among patients with acute metabolism-related neurological processes, the most common abnormality was reversible posterior encephalopathy syndrome, especially in those with hepatic encephalopathy, posttransplant patients, and patients with hypertensive emergencies.

Thirty-three of 129 (26\%) patients had normal or noncontributory findings on CT scans, but significant findings on MRI

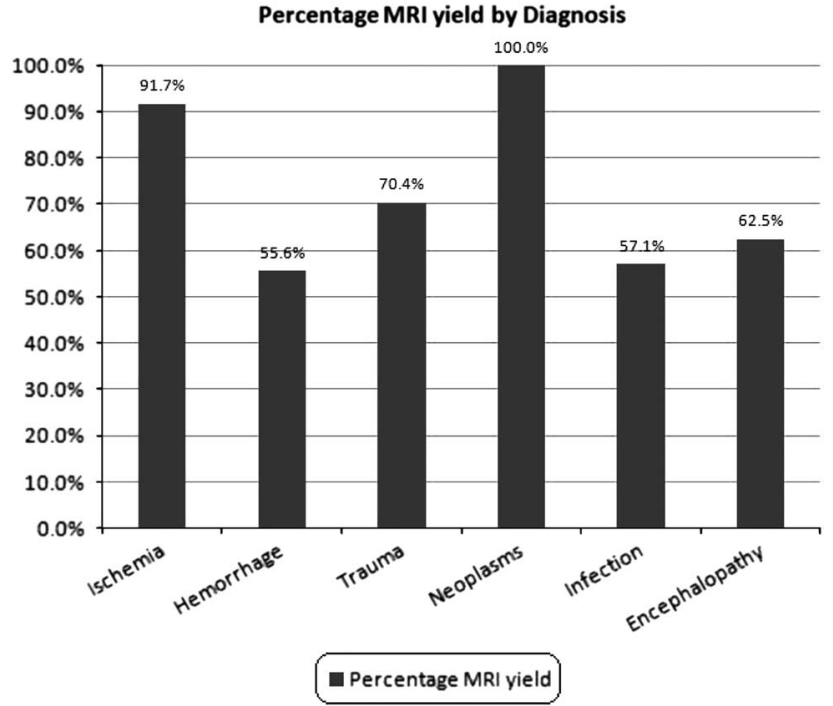

Figure 1: Additional diagnostic sensitivity of MRI over CT (MRI yield) across six diagnostic categories.

that were not only diagnostically helpful, but important in terms of predicting prognosis and considering the withdrawal of life support in certain critically ill patients who remained in a coma. For example, two patients had cardiac arrest and were unconscious with normal CT scans, but their MRI demonstrated increased signal on T2-weighted images, decreased signal on T1-weighted images, and restricted diffusion involving predominately the cortical and subcortical regions of both cerebral hemispheres, consistent with global ischemia. Four patients with traumatic brain injury had no significant findings on CT scan, but their MRI demonstrated diffuse axonal injury primarily involving the brain stem, associated with a very poor anticipated neurological outcome. Meanwhile, five patients had extensive bilateral strokes on MRI that were not seen on CT scan; these were also helpful for estimating prognosis.

Figures 2-4 provide examples of cases in which MRI findings clearly expanded upon those of CT. These include two patients with intracranial hemorrhages in whom MRI revealed an area of infarction around the hemorrhagic lesion that was not detected on CT (Figures 2 and 3); a comatose patient post-cardiac arrest in whom CT was normal, but MRI revealed diffusion restriction of both cerebral hemispheres and the left cerebellum (Figure 4).

Pearson $\chi 2$ analysis revealed a statistically significant difference in the rate of MRI findings not identified on CT across the six diagnostic groups $(\chi 2=15.84, p=0.007)$. Odds ratios were calculated for each diagnostic pair. When the lowest rate-which was the $55.6 \%$ rate identified for hemorrhagic lesions-was assigned as the reference value, odds ratios ranged from 8.80 (95\% CI 2.16-35.85) for ischemic lesions to 1.07 (0.29-3.90) for infection. A clear delineation was identified, with the rates for both ischemic and neoplastic lesions statistically higher than any of the rates for the four other diagnostic categories, which in turn did not differ amongst themselves. All odds ratios are presented in Table 1.

Power calculations estimating required sample sizes per category and overall, first to achieve 30 missed or underestimated lesions per category and second to identify 30 subjects overall who have management significantly altered by MRI findings are 
A

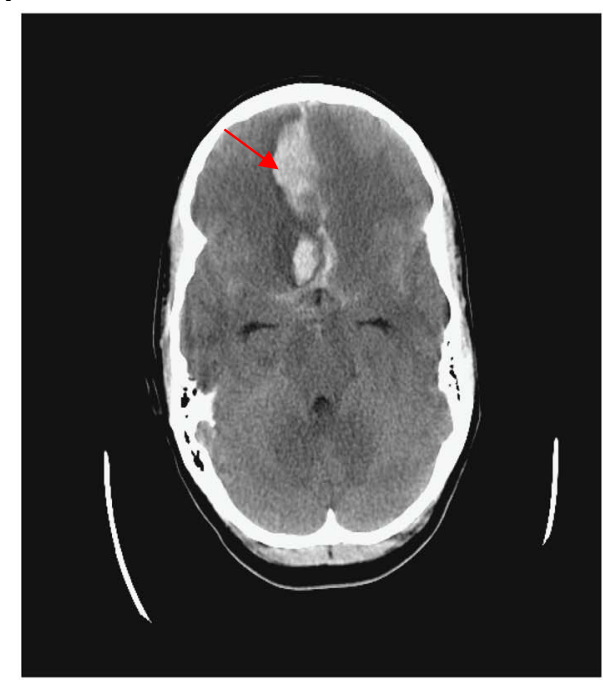

C

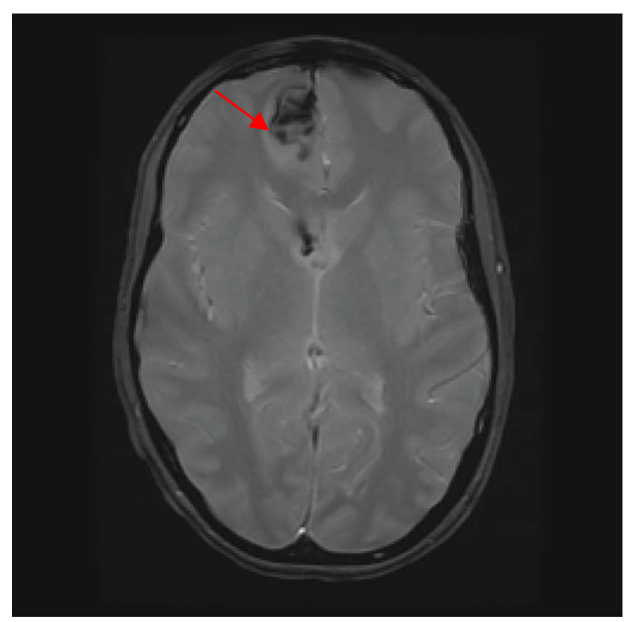

B

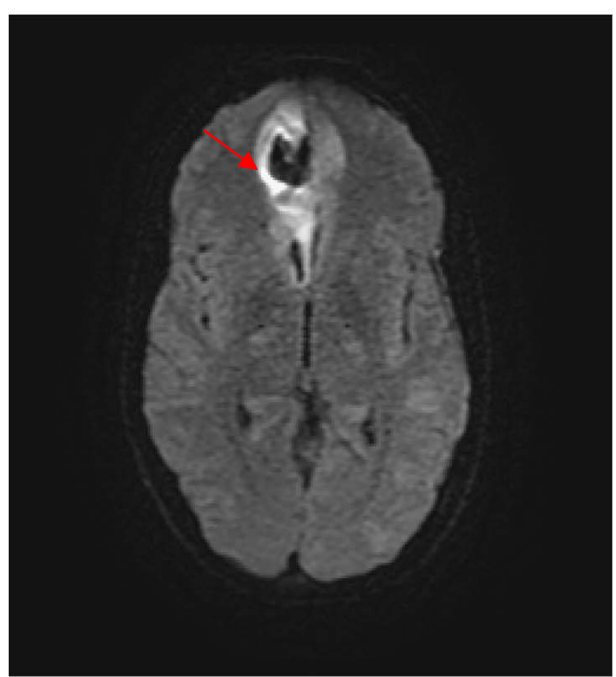

D

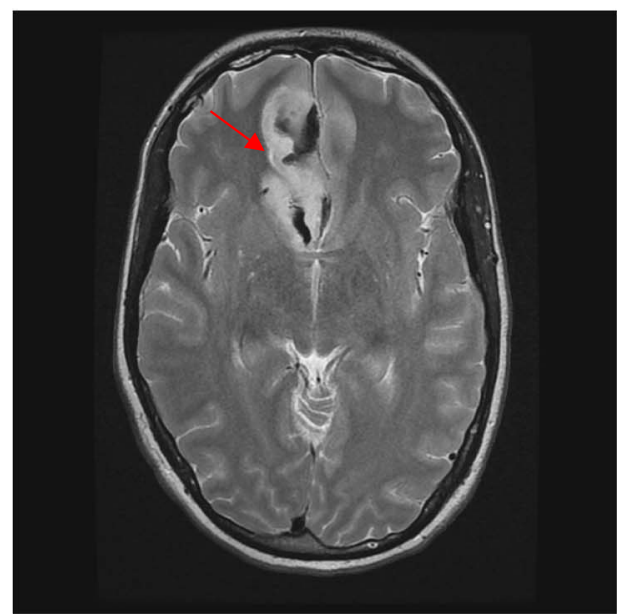

Figure 2: (A) CT of a patient with subarachnoid and intracerebral hemorrhage and MRI of the same patient $D W I(B)$, proton density $(C), T 2(D)$ showed infarction around the hemorrhagic lesion that was not seen on $C T$.

given in Table 2. Note that 129 subjects had 135 lesions (seven of the original 136 had no lesions, hence 129 subjects), and that the six subjects with two lesions all had a combination of ischemia and hemorrhage. This overlap has been adjusted for in the sample size estimates for these two categories.

\section{Discussion}

Considerable variation exists in the frequency with which various radiological services are used; this is particularly pronounced for MRI scans of the brain. Comparing rates of use across counties in Norway, for example, whereas the ratios of the highest to lowest rates of use were just 1.9 for radiographs of the chest and 2.7 for CT scans of the head/brain, the ratio for brain MRIs was 32.4. ${ }^{19}$ In fact, among 31 different images compared, this high-tolow ratio was surpassed only by the ratios for MRI of the knee (304.1), MRI of the cervical spine (139.0), and pelvic ultrasound (86.7), with standard X-rays of the lumbar spine (14.4) being the only other image for which the ratio was 10.0 or greater. ${ }^{19}$
For the initial evaluation of patients presenting with any neurological decline, CT scans have long been considered a costeffective neuroimaging method. ${ }^{4,18,19}$ Advantages of CT are that it is quick to perform, easily tolerated, and reliable at detecting certain brain lesions, particularly intracranial hemorrhages. However, some lesions can be subtle and, as such, difficult to detect on CT. Examples include acute ischemic strokes, especially lacunar strokes and strokes involving the brainstem. ${ }^{10-13,22,23}$ For these lesions, research has consistently demonstrated MRI to be superior to CT. ${ }^{10-13,22-24}$ For ischemic lesions, this is especially true with certain newer MRI techniques such as diffusionweighted imaging (DWI) and susceptibility-weighted imaging, with sensitivity rates for DWI as high as $98 \%$, versus just $30-70 \%$ for CT. ${ }^{12,13}$ However, it holds true even for conventional MRI versus CT and for very minor ischemic events. For example, in a just-published study of 168 patients with transient ischemic attacks and another 147 with minor strokes, standard MRI detected lesions in $39 \%$ and $86 \%$, versus just $8 \%$ and $18 \%$ with CT, respectively. $^{11}$ 

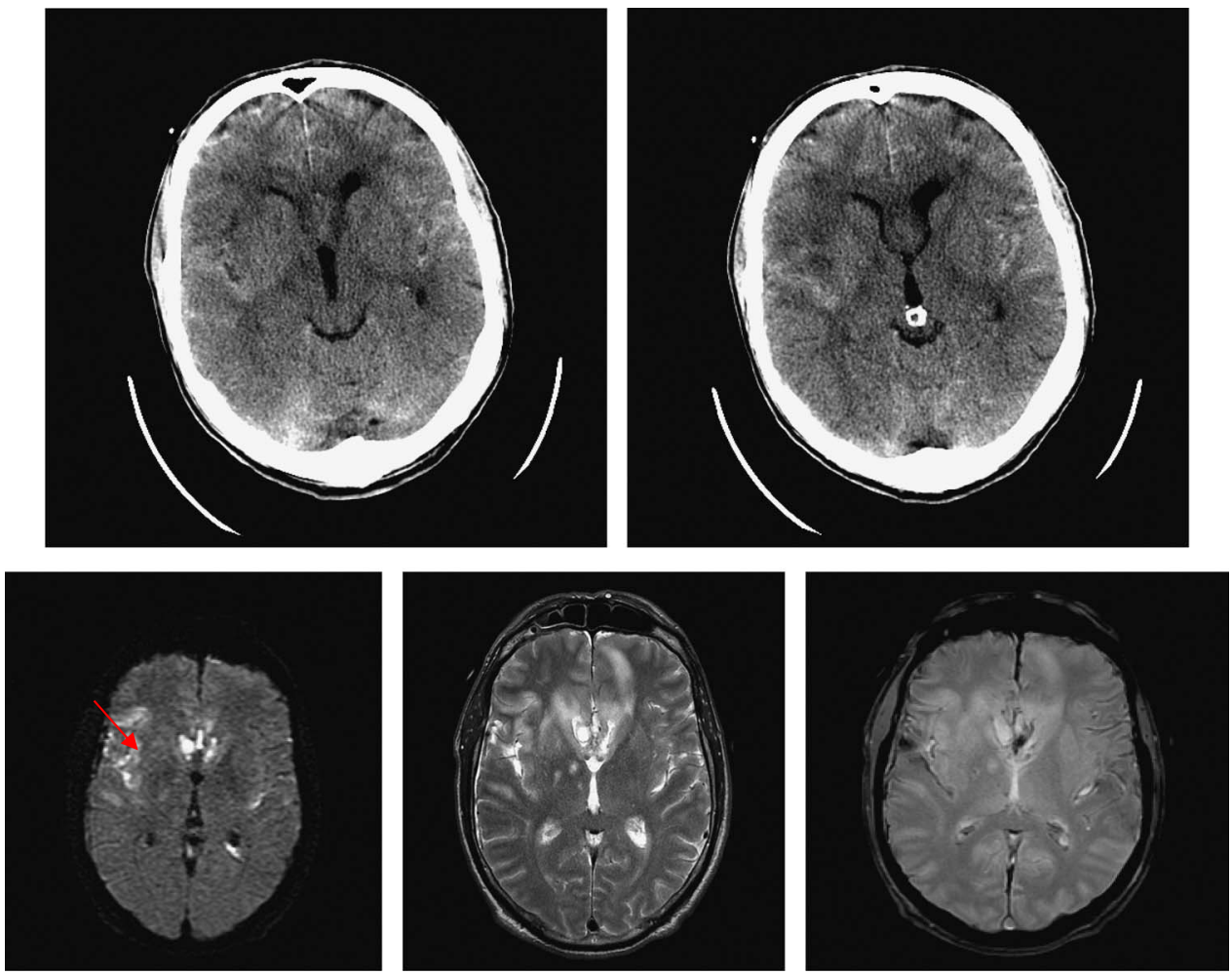

Figure 3: (A) CT of a patient with subarachnoid hemorrhage. (B) MRI of the same patient in Figure 3A, diffusion-weighted images, T2, and proton density images, respectively. DWI revealed a right frontal infarct not detected by CT (note the difference between MRI and CT scans cuts because of tilt angle and bone artifact).

MRI has been increasingly used in the initial assessment of patients with certain neurological disorders because it is more sensitive than CT at detecting and assessing the majority of abnormalities that may occur in patients with acute ischemic strokes or traumatic brain injuries and at identifying lesions within the mesencephalon or posterior fossa. ${ }^{23,25,26}$ Furthermore, MRI with DWI may provide information with respect to the chronicity of a lesion. One particular area where this is gaining increased attention is in the postoperative assessment of patients who have undergone especially cardiac but also vascular procedures that place them at high risk of ischemic brain events, silent or otherwise. ${ }^{27-32}$ The advantage of MRI over CT is especially noted with certain forms of infarct, such as lacunar and posterior fossa/ brainstem lesions. ${ }^{9,12,14,23,24}$ MRI also surpasses CT in terms of assessing the stage and evolution of postischemic hemorrhages. ${ }^{24,33} \mathrm{~A}$ further advantage specifically of DWI-MRI is that it can potentially discriminate between cytotoxic and vasogenic edema, ${ }^{34,35}$ which has potential relevance as an indicator of whether ischemic lesions will remain focal or become more global, and hence as an indicator of prognosis, especially in those who are clinically unresponsive, for whom any cognitive assessment is impossible. ${ }^{36}$ The numerous documented advantages of MRI over CT in the detection of brain ischemia was certainly supported by our series, which involved 136 patients overall and 36 with ischemic lesions, with more than $90 \%$ of the MRIs performed detecting clinically relevant findings beyond what had been observed on CT. In addition, in every one of our six patients in which both hemorrhagic and ischemic lesions were observed, there were findings on MRI not observed on CT, including ischemia in the setting of hemorrhage and vice versa.

The advantages of MRI over CT extend to nonischemic lesions as well. Chihangiroglu et $\mathrm{al}^{4}$ performed a detailed review, analyzing more than 20,000 radiological studies on patients older than age 60 with brain injury and found that brain MRI was superior to brain CT for detecting and classifying all forms of extra-axial hemorrhage (epidural, subdural, subarachnoid, and intraventricular); at identifying and grading diffuse axonal injuries, cortical contusions, and brain stem injuries; and at detecting secondary effects of trauma, including cerebral herniation, diffuse cerebral edema, vascular complications, and intracranial metabolic changes. ${ }^{4}$ In fact, the only area in which CT either outperformed or was equal to MRI was in detecting skull fractures. That being said, Wang et al recently reported that MRI cisternography outperformed CT in the detection of cerebrospinal leakage in patients with skull base fractures. ${ }^{3}$ In our series, $70 \%$ of the MRIs performed on patients with head trauma revealed findings not seen on CT, placing traumatic lesions at the mid-point between ischemic and neoplastic lesions (for which MRI yielded the highest rate of additional findings) and metabolic, infectious, and hemorrhagic lesions (for which MRI yielded the lowest rate, but still always more than 50\%).

Other major advantages of MRI over CT in the workup of patients with unknown but suspected brain abnormalities is its ability to provide, in addition to superior morphological data, a wide variety of further information through techniques such magnetic resonance angiography, diffusion, susceptibility- and 

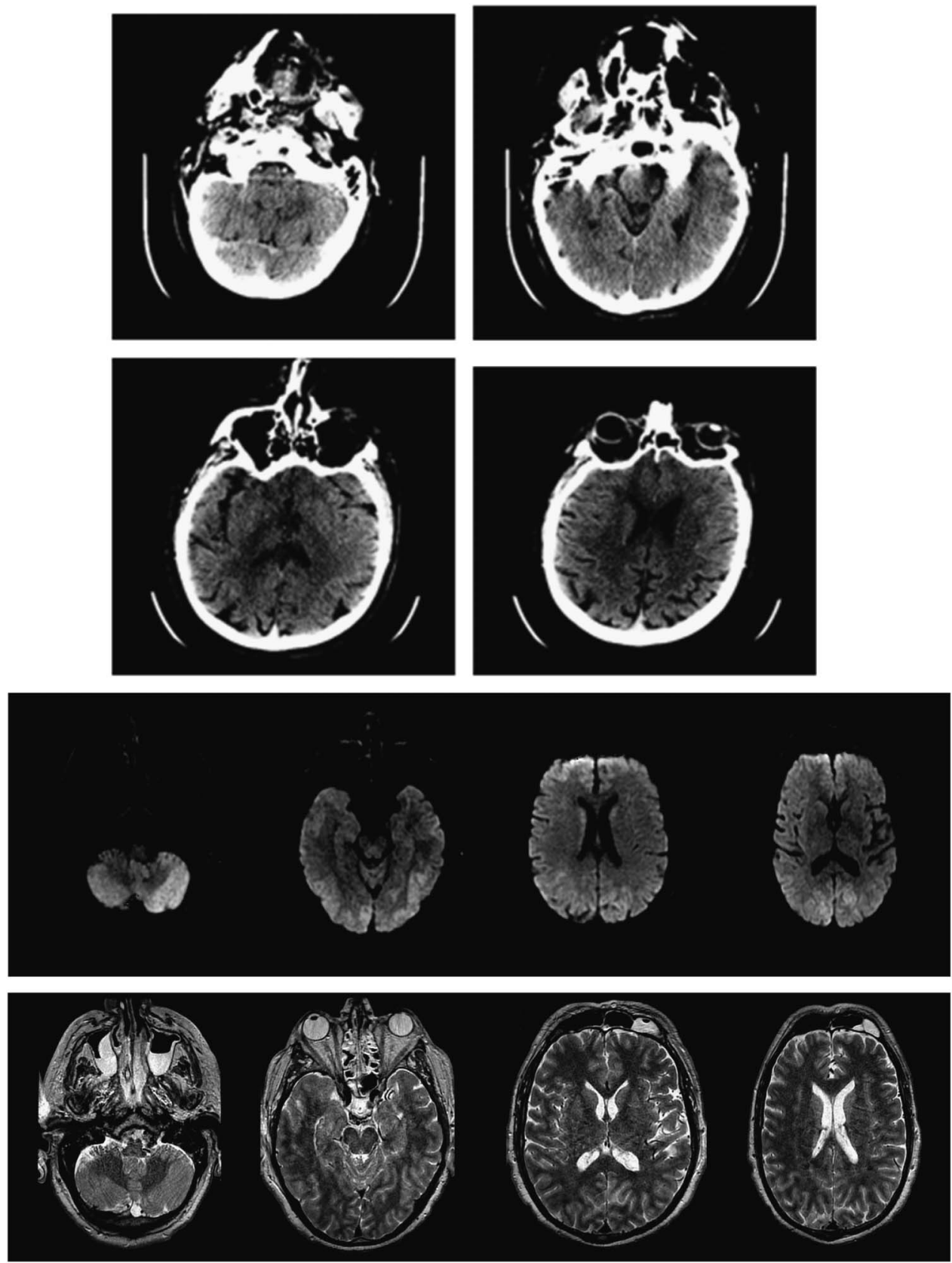

Figure 4: (A) Normal CT images of a patient who remained comatose after a cardiac arrest. MRI of the same patient as in Figure 4A; DWI and T2 images respectively demonstrating diffusion restriction of both cerebral hemispheres and the left cerebellum as seen on DWI.

perfusion-weighted images (e.g. DWI, functional MRI, and spectroscopy. ${ }^{36-38}$ With the routine use of electroencephalograms in most ICUs, MRI provides an even further advantage if the electrodes are made of metallic particles and, therefore, MRI-compatible. ${ }^{39}$ MRI is also a better tool to demonstrate contrast enhancement of either the parenchyma or meninges. ${ }^{40}$ As such, MRI allows for the detection of subtle findings of disorders such as focal cerebritis, encephalitis, and metabolic syndromes. ${ }^{17}$ In our study, MRI findings were diagnostic for reversible posterior fossa encephalopathy syndrome in a patient who had had an entirely normal CT.
Overall, across our sample of 136 patients, MRI revealed additional, clinically unsuspected pathology and/or better delineation of lesions identified on CT in 85 (63\%) patients. However, the MRI findings changed the working diagnosis (initially based on CT scans and/or clinical examination) in only six of 136 patients (4\%). The question that remains to be asked, then, is how the additional use of MRI influences treatment, outcomes, and costs. This is a question that clearly would be best addressed within the context of a large-scale prospective trial. The precedence for MRI actually altering treatment has already been established in other settings besides the ICU, most notably in the treatment of cancer, when the 
Table 1: Odds ratios comparing rates of new MRI findings not observed on CT between the six diagnostic groups

\begin{tabular}{|c|c|c|c|c|c|c|}
\hline Diagnosis & Hemorrhage & Ischemia & Trauma & Neoplasm & Infection & Metabolic encephalopathy \\
\hline \multirow[t]{2}{*}{ Hemorrhage } & 1.00 & 0.11 & 0.53 & 0.56 & 0.93 & 0.75 \\
\hline & & (0.03-0.46) & $(0.17-1.61)$ & $(0.40-0.78)$ & $(0.26-3.45)$ & $(0.24-2.33)$ \\
\hline Ischemia & $(2.16-35.95)$ & & $(1.10-20.00)$ & $(0.83-1.01)$ & $(1.69-40.00)$ & $(1.56-27.78)$ \\
\hline Trauma & 1.90 & 0.22 & 1.00 & 0.70 & 1.79 & 1.43 \\
\hline Neoplasm & $(1.29-2.53)$ & $(0.99-1.20)$ & (1.11-1.82) & & $(1.11-2.76)$ & $(1.17-2.18)$ \\
\hline \multirow[t]{2}{*}{ Infection } & 1.07 & 0.12 & 0.56 & 0.57 & 1.00 & 0.80 \\
\hline & $(0.29-3.90)$ & $(0.03-0.59)$ & $(0.14-2.20)$ & $(0.36-0.90)$ & & $(0.21-3.03)$ \\
\hline Metabolic & 1.33 & 0.15 & 0.70 & 0.63 & 1.25 & 1.00 \\
\hline
\end{tabular}

Odds ratios statistically different than 1.00 enlarged and in bold font.

Table 2: Rates of new findings on MRI vs. CT, and power calculations for sample size estimates to detect 30 such patients per diagnostic group

\begin{tabular}{|c|c|c|c|c|c|c|}
\hline $\begin{array}{l}\text { Diagnostic } \\
\text { category }\end{array}$ & Total lesions $\mathbf{N}=$ & New findings on MRI & $\begin{array}{c}\text { Percentage additional } \\
\text { yield }\end{array}$ & $95 \%$ confidence limits & $\begin{array}{l}\text { N required for larger } \\
\text { study ( } 0 \% \text { dropout) }\end{array}$ & $\begin{array}{l}\mathrm{N} \text { required for larger } \\
\text { study ( } 25 \% \text { dropout) }\end{array}$ \\
\hline Ischemia* & 36 & 33 & $91.7 \%$ & $76.4-97.8 \%$ & 40 & 53 \\
\hline Hemorrhage* & 27 & 15 & $55.6 \%$ & $35.6-74.0 \%$ & 66 & 88 \\
\hline Trauma & 27 & 19 & $70.4 \%$ & $49.7-84.2 \%$ & 43 & 57 \\
\hline Neoplasms & 7 & 7 & $100.0 \%$ & $56.1-100.0 \%$ & 30 & 40 \\
\hline Infection & 14 & 8 & $57.1 \%$ & $29.7-81.2 \%$ & 53 & 70 \\
\hline Encephalopathy & 24 & 15 & $62.5 \%$ & $40.8-80.5 \%$ & 48 & 64 \\
\hline All diagnoses & 135 & 97 & $71.9 \%$ & $63.4-79.1 \%$ & 279 & 372 \\
\hline $\begin{array}{l}\text { Diagnostic } \\
\text { category }\end{array}$ & Total lesions $\mathrm{N}=$ & Rx changed by MRI & Percentage additional yield & $95 \%$ confidence limits & $\begin{array}{l}\mathrm{N} \text { required for larger } \\
\text { study (no dropouts) }\end{array}$ & $\begin{array}{l}\mathrm{N} \text { required for larger } \\
\text { study ( } 25 \% \text { dropout) }\end{array}$ \\
\hline All diagnoses & 135 & 6 & $4.4 \%$ & $1.8-9.8 \%$ & 675 & 900 \\
\hline
\end{tabular}

*Note that 6 patients had BOTH an ischemic and hemorrhagic lesion

detection of additional lesions or specific lesion characteristics may significantly alter the nature of treatment given. For example, Muller-Horvat et al found that findings on whole-body MRI versus whole-body CT significantly influenced the choice of treatment in roughly one in four patients with metastatic melanoma. ${ }^{41}$ Meanwhile, Yokoi et al found that $50 \%$ versus less than $10 \%$ of brain metastases were detected preoperatively by MRI and CT, respectively, in patients potentially undergoing surgical resections for small cell cancer of the lung. ${ }^{16}$

Similar to patients with metastatic cancer, sometimes a decision must be made in the ICU as to how aggressive to be with treatment. In many patients in the ICU with severe brain injuries, the long-term prognosis is poor, both for survival and neurological recovery. ${ }^{42-44}$ As such, for reasons that include ethics, in this group of patients it would be especially helpful if long-term prognosis could be predicted reliably and relatively early in the course of assessment and treatment. In an attempt to predict clinical outcomes in these critically ill patients, both positive and negative results of diffusion and perfusion-weighted images may be of extreme use because they can suggest whether critical areas of the brain have been affected by a pathological process and to what extent. Changes in these areas may still be occult on CT or even with conventional MRI. ${ }^{36}$ All the patients in our study who were unconscious from circulatory or respiratory failure, as well as our one patient with suspected hepatic encephalopathy, demonstrated changes consistent with global ischemia, changes that were not apparent on CT scans. We also identified a comatose patient after cardiac arrest in whom CT was normal, but MRI revealed diffusion restriction of both cerebral hemispheres and the left cerebellum; and a patient in coma after a prolonged seizure in whom CT again was normal, but MRIs revealed diffuse ischemic changes bilaterally. In both these cases, the additional information provided by MRI affected management decisions regarding the aggressiveness of therapy.

A further issue that warrants mention is the issue of practicality. Critically ill ICU patients require respiratory support and 
continuous monitoring and are difficult to transport to MRI and CT scanners. With dedicated teams of ICU and MRI personnel working together, we did not encounter any safety concerns while scanning these patients, especially with the presence of MRI-compatible monitors and ventilators. However, should transferring such patients to the radiology department be one trip, rather than two, as is necessary when MRI is required because of nondiagnostic CT findings? The current study demonstrates that, in at least a subset of ICU patients at a single institution who ultimately required both imaging procedures, MRI was vastly more sensitive than CT at detecting and delineating lesions. Three critical questions for further study clearly are: (1) For what percentage of ICU patients with brain pathology and decreased consciousness does this apply? Clearly, for example, there may be a large percentage of patients for whom the initial CT scan is adequate; but what is this percentage? And what percentage of patients will not be eligible for MRI scanning, either because they are too agitated or too unstable? (2) Does this influence patient outcomes, including survival, morbidity rates, quality of life, and decisions to treat versus palliate? And (3) does this affect patient management costs and, if so, in what direction and to what extent? It is undeniable that MRI scans are more costly than CT scans. ${ }^{18}$ But if the additional information obtained by MRI leads to more directed and, therefore, more cost-effective management, including perhaps the withdrawal of aggressive management in patients found to have profound and likely irreversible brain injury, then cost savings might be an end result.

The current study has numerous admitted limitations, as is to be expected in a retrospective study for which very limited inferential testing was intended. These limitations include its retrospective nature and the sometimes prolonged period between the CT scans and MRI. Because MRI always was conducted later than $\mathrm{CT}$, it is likely that some of the information obtained by MRI could have been detected on CT if a follow-up CT had been performed instead of the MRI. This being said, some MRI-specific information, such as diffusion, cannot be obtained from CT. Moreover, almost $75 \%$ of the patients underwent their MRI within four days ( 96 hours) of their CT; and the clear majority of the remainder within 10 days. Given that MRI findings surpassed those of CT in almost two-thirds of patients, MRI findings were of added value in a sizeable percentage of those reexamined within four to ten days. A prospective study clearly is needed to clarify the time point until which MRI is better than follow-up CT, which again argues for the need for a larger, prospective clinical trial.

Another study limitation was the very small number $(n=7)$ of neoplastic lesions identified in our sample. This being said, despite this small number, there was a statically significant increased advantage of MRI over CT in patients with neoplasms versus patients with trauma, infections, metabolic-related abnormalities, and intracranial hemorrhages. In fact, in all seven patients, MRI yielded additional findings of clinical value, suggesting that there may be a very real and sizeable added value in this subset of patients.

\section{Conclusions}

In ICU patients with impaired brain function, there may be discordance between CT findings and clinical status. MRI provides significant new information over CT scans that often is useful at guiding management and determining outcome.
MRI clearly is superior to CT in at least a select population of ICU patients for answering clinical questions, an observation that certainly warrants further study to determine how often, to what extent, and how this influences outcomes and costs. Our results suggest that such a study is, in fact, feasible, and that patients with a broad range of pathological processes should be incorporated, including especially ischemic and neoplastic lesions, but also brain trauma, hemorrhagic lesions, infections, and metabolicrelated abnormalities. Hopefully, such a trial will be under way within the very near future.

\section{ACKNOWLEDGEMENTS}

The study was conducted at London Health Sciences Center, Western University, London, Ontario, Canada.

\section{Disclosures}

The authors have no disclosures to report.

\section{REFERENCES}

1. Bigler ED, Ryser DK, Gandhi P, Kimball J, Wilde EA. Day-of-injury computerized tomography, rehabilitation status, and development of cerebral atrophy in persons with traumatic brain injury. Am J Phys Med Rehabil. 2006;85:793-806.

2. Newton MR, Greenwood RJ, Britton KE, et al. A study comparing SPECT with CT and MRI after closed head injury. J Neurol Neurosurg Psychiatry. 1992;55:92-4.

3. Wang $\mathrm{X}, \mathrm{Xu} \mathrm{M}$, Liang $\mathrm{H}, \mathrm{Xu} \mathrm{L}$. Comparison of $\mathrm{CT}$ and MRI in diagnosis of cerebrospinal leak induced by multiple fractures of skull base. Radiol Oncol. 2011;45:91-6.

4. Cihangiroglu M, Ramsey RG, Dohrmann GJ. Brain injury: analysis of imaging modalities. Neurol Res. 2002;24:7-18.

5. Duhem R, Vinchon M, Tonnelle V, Soto-Ares G, Leclerc X. [Main temporal aspects of the MRI signal of subdural hematomas and practical contribution to dating head injury]. [In French] Neurochirurgie. 2006;52:93-104.

6. Kidwell CS, Chalela JA, Saver JL, et al. Comparison of MRI and CT for detection of acute intracerebral hemorrhage. JAMA. 2004;292:1823-30.

7. Renou P, Sibon I, Tourdias T, et al. Reliability of the ECASS radiological classification of postthrombolysis brain haemorrhage: a comparison of $\mathrm{CT}$ and three MRI sequences. Cerebrovasc Dis. 2010;29:597-604.

8. Yuan MK, Lai PH, Chen JY, et al. Detection of subarachnoid hemorrhage at acute and subacute/chronic stages: comparison of four magnetic resonance imaging pulse sequences and computed tomography. J Chin Med Assoc. 2005;68:131-7.

9. Davis SM, Tress BM, Dowling R, Donnan GA, Kiers L, Rossiter SC. Magnetic resonance imaging in posterior circulation infarction: impact on diagnosis and management. Aust $\mathrm{N} \mathrm{Z} \mathrm{J} \mathrm{Med.}$ 1989;19:219-25.

10. Lansberg MG, Albers GW, Beaulieu C, Marks MP. Comparison of diffusion-weighted MRI and CT in acute stroke. Neurology. 2000;54:1557-61.

11. Moreau F, Asdaghi N, Modi J, Goyal M, Coutts SB. Magnetic resonance imaging versus computed tomography in transient ischemic attack and minor stroke: the more you see the more you know. Cerebrovasc Dis Extra. 2013;3:130-6.

12. Smajlovic D, Sinanovic O. Sensitivity of the neuroimaging techniques in ischemic stroke. Med Arh. 2004;58:282-4.

13. Urbach H, Flacke S, Keller E, et al. Detectability and detection rate of acute cerebral hemisphere infarcts on CT and diffusionweighted MRI. Neuroradiology. 2000;42:722-7.

14. Engelter ST, Wetzel SG, Bonati LH, Fluri F, Lyrer PA. The clinical significance of diffusion-weighted MR imaging in stroke and TIA patients. Swiss Med Wkly. 2008;138:729-40. 
15. Suwanwela N, Tantanatrakool B, Suwanwela NC. Intracranial lymphoma: CT and MR findings. J Med Assoc Thai. 2001;84: S228-43.

16. Yokoi K, Kamiya N, Matsuguma H, et al. Detection of brain metastasis in potentially operable non-small cell lung cancer: a comparison of CT and MRI. Chest. 1999;115:714-9.

17. Leira HL, Myhr G, Nilsen G, Dale LG. Cerebral magnetic resonance imaging and cerebral computerized tomography for patients with solvent-induced encephalopathy. Scand J Work Environ Health. 1992;18:68-70.

18. Ibrahim R, Samion S, Mazli MZ, Amrizal MN, Aljunid SM. Cost of magnetic resonance imaging (MRI) and computed tomography (CT) scan in UKMMC. BMC. Health Serv Res. 2014;12:11-2.

19. Lysdahl KB, Borretzen I. Geographical variation in radiological services: a nationwide survey. BMC. Health Serv Res. 2007;15:21.

20. Bermingham $\mathrm{S}$. The appropriate use of neuroimaging in the diagnostic work-up of dementia: an economic literature review and cost-effectiveness analysis. Ont Health Technol Assess Ser. 2014;14:1-67.

21. Devore JL. Probability and Statistics for Engineering and the Sciences, 4th ed.. Duxbury Press; 1995.

22. Assouline E, Benziane K, Reizine D, et al. Intra-arterial thrombus visualized on $\mathrm{T} 2 *$ gradient echo imaging in acute ischemic stroke. Cerebrovasc Dis. 2005;20:6-11.

23. Brazzelli M, Sandercock PAG, Chappell FM, et al. Magnetic resonance imaging versus computed tomography for detection of acute vascular lesions in patients presenting with stroke symptoms (review). Cochrane Database Syst Rev., 2009:CD007424.

24. Saur D, Kucinski T, Grzyska U, et al. Sensitivity and interrater agreement of CT and diffusion-weighted MR imaging in hyperacute stroke. Am J Neuroradiol. 2003;24:878-85.

25. Gentry LR, Godersky JC, Thompson B, Dunn VD. Prospective comparative study of intermediate-field MR and CT in the evaluation of closed head trauma. Am J Roentgenol. 1988;150:673-82.

26. Kent DL, Haynor DR, Longstreth WT Jr, Larson EB. The clinical efficacy of magnetic resonance imaging in neuroimaging. Ann Intern Med. 1994;15:856-71.

27. Lovblad KO, Pluschke W, Remonda L, et al. Diffusion-weighted MRI for monitoring neurovascular interventions. Neuroradiology. 2000;42:134-8.

28. Palombo G, Faraglia V, Stella N, Giugni E, Bozzao A, Taurino M. Late evaluation of silent cerebral ischemia detected by diffusionweighted MR imaging after filter-protected carotid artery stenting. AJNR Am J Neuroradiol. 2008;29:1340-3.

29. Restrepo L, Wityk RJ, Grega MA, et al. Diffusion- and perfusionweighted magnetic resonance imaging of the brain before and after coronary artery bypass grafting surgery. Stroke. 2002;33:2915.

30. Rodes-Cabau J, Dumont E, Boone RH, et al. Cerebral embolism following transcatheter aortic valve implantation: comparison of transfemoral and transapical approaches. J Am Coll Cardiol. 2011;57:18-28.
31. Stolz E, Gerriets T, Kluge A, et al. Diffusion-weighted magnetic resonance imaging and neurobiochemical markers after aortic valve replacement: implications for future neuroprotective trials? Stroke. 2004;35:888-92.

32. Sun X, Lindsay J, Monsein LH, Hill PC, Corso PJ. Silent brain injury after cardiac surgery: a review: cognitive dysfunction and magnetic resonance imaging diffusion-weighted imaging findings. J Am Coll Cardiol. 2012;60:791-7.

33. Atlas SW, Thulborn KR. Intracranial hemorrhage. In Atlas SW ed. Magnetic Resonance Imaging of the Brain and Spine, 3rd ed. Philadelphia: Lippincott Williams and Wilkins; 2002:773-832.

34. Schaefer PW, Buonanno FS, Gonzalez R, Schwamm LH. Diffusion weighted imaging discriminates between cytotoxic and vasogenic edema in a patient with eclampsia. Stroke. 1997;28:1082-5.

35. Schwartz RB, Mulkern RV, Gudbjartsson H, Jolesz F. Diffusion weighted MR imaging in hypertensive encephalopathy: clues to pathogenesis. Am J Neuroradiol. 1988;19:859-62.

36. Sundgren PC, Reinstrup P, Romner B, Holtas S, Maly P. Value of conventional, and diffusion and perfusion weighted MRI in the management of patients with unclear cerebral pathology, admitted to the intensive care unit. Neuroradiology. 2002;44:674-80.

37. Gofton TE, Chouinard PA, Young GB, et al. Functional MRI study of the primary somatosensory cortex in comatose survivors of cardiac arrest. Exp Neurol. 2009;217:320.

38. Norton LE, Hutchinson RM, Young GB, Lee DH, Sharpe MD, Mirsattari SM. Disruptions of functional connectivity in the default mode network of comatose patients. Neurology. 2012;78:175-81.

39. Mirsattari SM, Davies-Schinkel C, Young GB, Sharpe MD, Lee DH. Usefulness of a 1.5 tesla MRI-compatible EEG electrode system for routine use in the intensive care unit of a tertiary care hospital. Epilepsy Res. 2009;84:28-32.

40. Yildirim Donmez F, Guvenc Z, Emiroglu FK, Coskun M, Haberal M. Evaluation of neurological complications in pediatric liver transplant recipients: MRI versus CT. J Child Neurol. 2009;24:656.

41. Muller-Horvat C, Radny P, Eigentler TK, et al. Prospective comparison of the impact on treatment decisions of whole-body magnetic resonance imaging and computed tomography in patients with metastatic malignant melanoma. Eur J Cancer. 2006;42:342-50.

42. Calvi MR, Beretta L, Dell'Acqua A, Anzalone N, Licini G, Gemma M. Early prognosis after severe traumatic brain injury with minor or absent computed tomography scan lesions. J Trauma. 2011;70: 447-51.

43. Pierallini A, Pantano P, Fantozzi LM, et al. Correlation between MRI findings and long-term outcome in patients with severe brain trauma. Neuroradiology. 2000;42:860-7.

44. Skandsen T, Kvistad KA, Solheim O, Strand IH, Folvik M, Vik A. Prevalence and impact of diffuse axonal injury in patients with moderate and severe head injury: a cohort study of early magnetic resonance imaging findings and 1-year outcome. J Neurosurg. 2010;113:556-63. 